\title{
\begin{tabular}{l|l} 
Mibraries & DSpace@MIT
\end{tabular}
}

\author{
MIT Open Access Articles
}

\section{A Contraction Theory Approach to Singularly Perturbed Systems with Application to Retroactivity Attenuation}

The MIT Faculty has made this article openly available. Please share how this access benefits you. Your story matters.

Citation: Del Vecchio, Domitilla, and Jean-Jacques Slotine. "A Contraction Theory Approach to Singularly Perturbed Systems with Application to Retroactivity Attenuation." IEEE, 2011. 58315836.

As Published: http://dx.doi.org/10.1109/CDC.2011.6160340

Publisher: Institute of Electrical and Electronics Engineers (IEEE)

Persistent URL: http://hdl.handle.net/1721.1/79864

Version: Author's final manuscript: final author's manuscript post peer review, without publisher's formatting or copy editing

Terms of use: Creative Commons Attribution-Noncommercial-Share Alike 3.0 


\title{
A Contraction Theory Approach to Singularly Perturbed Systems with Application to Retroactivity Attenuation
}

\author{
Domitilla Del Vecchio and Jean-Jacques Slotine
}

\begin{abstract}
In this paper, we revisit standard results for singularly perturbed systems on the infinite time interval by employing tools from nonlinear contraction theory. This allows us to determine explicit bounds both on the rate of convergence of trajectories to the slow manifold, and on the distance between these trajectories and those of the reduced system. We illustrate the application of the proposed technique to the problem of retroactivity attenuation in biomolecular systems, that is, to the problem of attenuating the effects of output loading due to interconnection to downstream systems. By virtue of the explicit bounds, we can single out the key biochemical parameters to tune in order to enhance retroactivity attenuation. This provides design guidelines for synthetic biology devices that are robust to loading and can function as insulation devices just like insulating amplifiers work in electronics.
\end{abstract}

\section{INTRODUCTION}

Multiple time-scales have been viewed as a key ingredient of the modular architecture of complex systems ever since [23]. In recent years, this perspective has been strengthened in the context of the flurry of research in systems biology, most notably by $[11,13]$. A mathematical formulation in the context of interconnections between biomolecular networks has been provided by $[5,9,10]$. In these works, timescale separation was shown to be an effective mechanism to provide dynamic insulation between components and to enforce modular behavior. Modular behavior is particularly appealing as it guarantees that a system input/output response is not affected by interconnection to other systems. This allows to easily predict the behavior of a large system from the behavior of its subsystems. Modular behavior in the context of biomolecular systems is especially important in synthetic biology, in which researchers are engineering large networks starting from small working circuits [2].

Mathematically, the standard description of dynamical systems with multiple time-scales is based on singular perturbation theory, whose main results were established more than 40 years ago $[12,15]$. The main results state that the trajectories of the system fast approach an $\epsilon$-neighbor of the slow manifold, in which $\epsilon$ quantifies the ratio between slow and fast time scales. Convergence results on the finite time interval require local exponential stability of the slow manifold, while results on the infinite time interval also require exponential stability of the equilibrium point of the reduced system.

In this paper, we use comparatively recent convergence analysis tools, based on nonlinear contraction theory and

The authors are with Faculty of Mechanical Engineering, MIT, Cambridge, USA. This work was in part supported by NSF-CCF grant number 1058127 ddv@mit.edu virtual dynamical systems, to revisit some results on singular perturbation. Nonlinear contraction theory [18,25], a viewpoint on incremental stability which we briefly review in Section II, has emerged as a powerful tool in applications ranging from Lagrangian mechanics to network control. Historically, ideas closely related to contraction can be traced back to [7] and even to [16] (see also [3,21], and e.g. [17] for a more exhaustive list of related references). In addition, contraction is preserved through a large variety of systems combinations, which may make it particularly suitable in the context of biological systems [11], subject to evolution and development mechanisms. Employing nonlinear contraction theory, we provide a global convergence result on the infinite time interval for singularly perturbed systems. Specifically, assuming that the reduced system and the fast system are each partially contracting, we give explicit bounds both on the convergence rate to the slow manifold and on the distance of the system trajectories from those of the reduced system.

Explicit bounds are particularly useful in design problems in which specific values of $\epsilon$ can be chosen to obtain a desired approximation of the system behavior on the slow manifold. This is the case, for example, of methods for retroactivity attenuation in biomolecular systems based on time scale separation as studied in $[9,10]$. In these works, it was shown that the interconnection structure between biomolecular systems is such that making the time scale of an upstream system sufficiently fast is an effective means for attenuating the retroactivity to the output due to loading effects from a downstream system. Here, we provide explicit bounds on the rate of attenuation and the amount of retroactivity attenuation as functions of system parameters and $\epsilon$. We illustrate the application of these tools to design an insulation system based on phosphorylation, which attenuates retroacitivity based on the fast time scales of the phosphorylation reactions.

This paper is organized as follows. In Section II, we review basic tools in contraction theory. In Section III, results on global convergence of singularly perturbed systems are provided along with explicit convergence bounds. In Section IV, we apply these tools to study modular interconnection in biomolecular systems. In Section V, we provide a concrete biomolecular system example.

\section{BASIC CONTRACTION THEORY TOOLS}

Recall that, given a norm $|\cdot|$ on the state space, and its induced matrix norm $\|\mathbf{A}\|$, for an arbitrary square matrix $\mathbf{A}$, the associated matrix measure $m$ is defined as (see [4], 
[20])

$$
m(\mathbf{A}):=\lim _{h \rightarrow 0^{+}} \frac{1}{h}(\|\mathbf{I}+h \mathbf{A}\|-1) .
$$

The basic result of nonlinear contraction analysis [18] which we shall use in this paper can be stated as follows.

Theorem 1 (Contraction): Consider the $m$-dimensional deterministic system

$$
\dot{\mathbf{x}}=\mathbf{f}(\mathbf{x}, t)
$$

where $f$ is a smooth nonlinear function. The system is said to be contracting if any two trajectories, starting from different initial conditions, converge exponentially to each other. A sufficient condition for a system to be contracting is the existence of some matrix measure, $m$, such that

$$
\exists \lambda>0, \forall \mathbf{x}, \quad \forall t \geq 0, \quad m\left(\frac{\partial \mathbf{f}(\mathbf{x}, t)}{\partial \mathbf{x}}\right) \leq-\lambda .
$$

The scalar $\lambda$ defines the contraction rate of the system.

The standard matrix measures are listed in Table I. More generally, contraction may be shown by using matrix measures induced by the weighted vector norm $|\mathbf{x}|_{\boldsymbol{\Theta}, i}=|\boldsymbol{\Theta} \mathbf{x}|_{i}$, with $\Theta$ a constant invertible matrix and $i=1,2, \infty$. Such measures, denoted with $m_{\boldsymbol{\Theta}, i}$, are linked to the standard measures by:

$$
m_{\boldsymbol{\Theta}, i}(\mathbf{A})=m_{i}\left(\mathbf{\Theta} \mathbf{A} \boldsymbol{\Theta}^{-1}\right), \quad \forall i=1,2, \infty .
$$

Note that for linear time-invariant systems, contraction is equivalent to strict stability, and, using the Euclidean vector norm, $\Theta$ can be chosen as the transformation matrix which diagonalizes the system or puts it in Jordan form [18]. More generally, contraction can use time-varying and statedependent $\boldsymbol{\Theta}(\mathbf{x}, t)$, where $\boldsymbol{\Theta}(\mathbf{x}, t)^{T} \boldsymbol{\Theta}(\mathbf{x}, t)$ is uniformly positive definite and the so-called generalized Jacobian

$$
\mathbf{F}=\boldsymbol{\Theta}\left(\frac{\partial \mathbf{f}(\mathbf{x}, t)}{\partial \mathbf{x}}\right) \boldsymbol{\Theta}^{-1}+\dot{\boldsymbol{\Theta}} \Theta^{-1}
$$

has a uniformly negative definite matrix measure [18].

TABLE I

Standard MATrix Measures For a REAL $n \times n$ MATriX, $\mathbf{A}=\left[a_{i j}\right]$. The $i$-Th EIGENVAlue of A IS DENOTED With $\lambda_{i}(\mathbf{A})$.

\begin{tabular}{|c|c|}
\hline vector norm, $|\cdot|$ & induced matrix measure, $m(\mathbf{A})$ \\
\hline$|\mathbf{x}|_{1}=\sum_{j=1}^{n}\left|x_{j}\right|$ & $m_{1}(\mathbf{A})=\max _{j}\left(a_{j j}+\sum_{i \neq j}\left|a_{i j}\right|\right)$ \\
\hline$|\mathbf{x}|_{2}=\left(\sum_{j=1}^{n}\left|x_{j}\right|^{2}\right)^{\frac{1}{2}}$ & $m_{2}(\mathbf{A})=\max _{i}\left(\lambda_{i}\left\{\frac{A+A^{*}}{2}\right\}\right)$ \\
\hline$|\mathbf{x}|_{\infty}=\max _{1 \leq j \leq n}\left|x_{j}\right|$ & $m_{\infty}(\mathbf{A})=\max _{i}\left(a_{i i}+\sum_{j \neq i}\left|a_{i j}\right|\right)$ \\
\hline
\end{tabular}

For convenience, we will also say that a function $\mathbf{f}(\mathbf{x}, t)$ is contracting if the system $\dot{\mathbf{x}}=\mathbf{f}(\mathbf{x}, t)$ satisfies the sufficient condition above. Similarly, we will then say that the corresponding Jacobian matrix $\frac{\partial f}{\partial x}(\mathbf{x}, t)$ is contracting. In the sequel, unless otherwise stated, norms are Euclidean norms.

We shall also use the following two properties of contracting systems, whose proofs can be found in $[18,24]$.
Lemma 1: (Robustness) Assume that the system

$$
\dot{\mathbf{x}}=\mathbf{f}(\mathbf{x}, t)
$$

is contracting, with an associated metric transformation $\Theta$ and contraction rate $\lambda$, and consider the "perturbed" system

$$
\dot{\mathbf{x}}_{p}=\mathbf{f}\left(\mathbf{x}_{p}, t\right)+\mathbf{d}\left(\mathbf{x}_{p}, t\right)
$$

where $\mathbf{d}\left(\mathbf{x}_{p}, t\right)$ is bounded, that is,

$$
\exists d \geq 0, \forall \mathbf{x}_{p}, \forall t \geq 0, \quad\left|\mathbf{d}\left(\mathbf{x}_{p}, t\right)\right| \leq d .
$$

Then, any trajectory of the perturbed system satisfies

$$
\left|\mathbf{x}_{p}(t)-\mathbf{x}(t)\right| \leq \chi e^{-\lambda t}\left|\mathbf{x}_{p}(0)-\mathbf{x}(0)\right|+\frac{d \chi}{\lambda},
$$

in which $\chi$ is an upper bound on the condition number of $\Theta$ and $k$ is the induced norm of $\Theta[18,19]$.

Proof: With $R(t)=\left|\boldsymbol{\Theta}\left(\mathbf{x}_{p}(t)-\mathbf{x}(t)\right)\right|$ [18], one has

$$
\frac{d}{d t} R+\lambda R \leq\left|\boldsymbol{\Theta d}\left(\mathbf{x}_{p}, t\right)\right| .
$$

\section{MAIN RESULT}

We revisit standard results on singular perturbation using convergence analysis tools based on nonlinear contraction theory and virtual dynamical systems.

We consider the standard singular perturbation framework [12]

$$
\begin{aligned}
\dot{\mathbf{x}} & =\mathbf{f}(\mathbf{x}, \mathbf{z}, t) \\
\epsilon \dot{\mathbf{z}} & =\mathbf{g}(\mathbf{x}, \mathbf{z}, \epsilon)
\end{aligned}
$$

with $\epsilon>0$ a constant, $\mathbf{x} \in D_{x} \subseteq \mathbb{R}^{n}$, and $\mathbf{z} \in D_{z} \subseteq \mathbb{R}^{m}$.

Definition 1: [25] System (4) is said to be partially contracting in $\mathrm{x}$ if the virtual system

$$
\dot{\mathbf{y}}_{x}=\mathbf{f}\left(\mathbf{y}_{x}, \mathbf{z}(t), t\right)
$$

is contracting for any $\mathbf{z}(t)$ and for all $t$. Similarly, system (4) is said to be partially contracting in $\mathbf{z}$ if the virtual system

$$
\epsilon \dot{\mathbf{y}}_{z}=\mathbf{g}\left(\mathbf{x}(t), \mathbf{y}_{z}, \epsilon\right)
$$

is contracting for any $\mathbf{x}(t)$ and for all $\epsilon>0$.

Proposition 1: If system (4) is partially contracting in $\mathbf{z}$, then equation

$$
\mathbf{g}(\mathbf{x}, \mathbf{z}, \epsilon)=\mathbf{0}
$$

can be equivalently written as $\mathbf{z}=\gamma(\mathbf{x}, \epsilon)$, i.e., there is a unique, global mapping between $\mathbf{x}, \epsilon$ and $\mathbf{z}$.

Proof: The virtual system

$$
\epsilon \dot{\mathbf{y}}_{z}=\mathbf{g}\left(\mathbf{x}_{o}, \mathbf{y}_{z}, \epsilon\right)
$$

is contracting by hypothesis, for any $\mathbf{x}_{o}(t)$. If we set $\mathbf{x}_{o}$ equal to some constant vector, this system is also autonomous, and therefore tends to a unique equilibrium [18]. Thus for any given $\mathbf{x}_{o}$, the algebraic equation $\mathbf{g}\left(\mathbf{x}_{o}, \mathbf{y}_{z}, \epsilon\right)=\mathbf{0}$ has a unique solution, which we can denote $\mathbf{y}_{z}=\gamma\left(\mathbf{x}_{o}, \epsilon\right)$. 
Denote $\gamma(\mathbf{x}):=\gamma(\mathbf{x}, 0)$ and assume it is globally differentiable. Differentiating the relation $\mathbf{g}(\mathbf{x}, \gamma(\mathbf{x}), 0)=\mathbf{0}$ with respect to $\mathbf{x}$ then yields

$$
\frac{\partial \gamma(\mathbf{x})}{\partial \mathbf{x}}=-\left(\frac{\partial \mathbf{g}}{\partial \mathbf{z}}\right)^{-1} \frac{\partial \mathbf{g}}{\partial \mathbf{x}}(\mathbf{x}, \gamma(\mathbf{x}), 0)
$$

which is valid globally, as the matrix $\frac{\partial g}{\partial z}$ is uniformly invertible. The set $\{(\mathbf{x}, \mathbf{z}) \mid \mathbf{z}=\gamma(\mathbf{x})\}$ is commonly referred to as the slow manifold.

Lemma 2: Assume that system (4) is partially contracting in $\mathbf{z}$, with an associated metric transformation $\boldsymbol{\Theta}_{z}$, and let $\lambda_{z} / \epsilon$ be the contraction rate of (6). Assume further that, given (7), one can write

$$
\exists d \geq 0, \forall \mathbf{x}, \forall \mathbf{z}, \forall t \geq 0, \quad\left|\frac{\partial \gamma(\mathbf{x})}{\partial \mathbf{x}} \mathbf{f}(\mathbf{x}, \mathbf{z}, t)\right| \leq d
$$

and that $\mathbf{g}(\mathbf{x}, \mathbf{z}, \epsilon)$ is Lipschitz in $\epsilon$ with constant $K$. Then, any trajectory of (4) is such that

$$
\begin{array}{r}
|\mathbf{z}(t)-\gamma(\mathbf{x}(t))| \leq \chi_{z} e^{-\left(\lambda_{z} / \epsilon\right) t}|\mathbf{z}(0)-\gamma(\mathbf{x}(0))|+ \\
\frac{(d+K) \chi_{z}}{\lambda_{z}} \epsilon \forall t \geq 0
\end{array}
$$

where $\chi_{z}$ is an upper bound on the condition number of $\boldsymbol{\Theta}_{z}$.

Proof: Note that $\mathbf{y}_{z}=\mathbf{z}(t)$ is a solution of the contracting virtual system

$$
\epsilon \dot{\mathbf{y}}_{z}=\mathbf{g}\left(\mathbf{x}(t), \mathbf{y}_{z}, \epsilon\right)
$$

while $\mathbf{y}_{z d}=\gamma(\mathbf{x}(t))$ is a solution of the "perturbed" contracting virtual system

$$
\begin{array}{r}
\epsilon \dot{\mathbf{y}}_{z d}=\mathbf{g}\left(\mathbf{x}(t), \mathbf{y}_{z d}, \epsilon\right)+\epsilon \frac{\partial \gamma(\mathbf{x})}{\partial \mathbf{x}} \mathbf{f}(\mathbf{x}(t), \mathbf{z}(t), t)+ \\
\left(\mathbf{g}\left(\mathbf{x}(t), \mathbf{y}_{z d}, 0\right)-\mathbf{g}\left(\mathbf{x}(t), \mathbf{y}_{z d}, \epsilon\right)\right) .
\end{array}
$$

Applying the basic robustness result (3) of Section 2 yields the bound (8).

Theorem 2: Assume, in addition to the hypotheses of the previous Lemma, that system (4) is partially contracting in $\mathbf{x}$, with an associated metric transformation $\boldsymbol{\Theta}_{x}$, and let $\lambda_{x}$ be the contraction rate of (5). Assume furthermore that $\mathbf{f}(\mathbf{x}, \mathbf{z}, t)$ is Lipschitz in $\mathbf{z}$, with Lipschitz constant $\alpha$ and that $\gamma(\mathbf{x})$ is Lipschitz with Lipschitz constant $\alpha_{\gamma}$. Let $\mathbf{x}_{\gamma}$ be a solution of the reduced system

$$
\dot{\mathbf{x}}_{\gamma}=\mathbf{f}\left(\mathbf{x}_{\gamma}, \gamma\left(\mathbf{x}_{\gamma}\right), t\right) .
$$

Then, any trajectory of (4) satisfies

$$
\begin{array}{r}
\left|\mathbf{x}(t)-\mathbf{x}_{\gamma}(t)\right| \leq \chi_{x}\left|\mathbf{x}(0)-\mathbf{x}_{\gamma}(0)\right| e^{-\lambda_{x} t}+ \\
\epsilon\left(C_{1}\left(e^{-\lambda_{x} t}-e^{-\left(\lambda_{z} / \epsilon\right) t}\right)+C_{2}\left(1-e^{-\lambda_{x} t}\right)\right), \forall t \geq 0,
\end{array}
$$

and

$$
\begin{array}{r}
\left|\mathbf{z}(t)-\gamma\left(\mathbf{x}_{\gamma}(t)\right)\right| \leq \chi_{z} e^{-\left(\lambda_{z} / \epsilon\right) t}|\mathbf{z}(0)-\gamma(\mathbf{x}(0))|+ \\
\frac{(d+K) \chi_{z}}{\lambda_{z}} \epsilon+\alpha_{\gamma} \chi_{x}\left|\mathbf{x}(0)-\mathbf{x}_{\gamma}(0)\right| e^{-\lambda_{x} t}+ \\
\alpha_{\gamma} \epsilon\left(C_{1}\left(e^{-\lambda_{x} t}-e^{-\left(\lambda_{z} / \epsilon\right) t}\right)+C_{2}\left(1-e^{-\lambda_{x} t}\right)\right), \forall t \geq 0,
\end{array}
$$

in which

$$
C_{1}=\frac{\chi_{x} \alpha \chi_{z}|\mathbf{z}(0)-\gamma(\mathbf{x}(0))|}{\left(\lambda_{z}-\epsilon \lambda_{x}\right)}, \quad C_{2}=\frac{\chi_{x} \alpha \chi_{z}(d+K)}{\lambda_{z} \lambda_{x}},
$$

and $\chi_{x}$ is an upper bound on the condition number of $\boldsymbol{\Theta}_{x}$.

Proof: Using bound (8), one can write $\dot{\mathbf{x}}=\mathbf{f}(\mathbf{x}, \mathbf{z}, t)=$ $\mathbf{f}\left(\mathbf{x}, \gamma(\mathbf{x})+\Delta_{1}(t), t\right)=\mathbf{f}(\mathbf{x}, \gamma(\mathbf{x}), t)+\Delta_{2}(t)$, where $\Delta_{2}(t)=\mathbf{f}\left(\mathbf{x}, \gamma(\mathbf{x})+\Delta_{1}(t), t\right)-\mathbf{f}(\mathbf{x}, \gamma(\mathbf{x}), t)$ and $\Delta_{1}(t)=$ $\chi_{z} e^{-\left(\lambda_{z} / \epsilon\right) t}|\mathbf{z}(0)-\gamma(\mathbf{x}(0))|+\frac{(d+K) \chi_{z}}{\lambda_{z}} \epsilon$. Since $\mathbf{f}$ is Lipschitz in $\mathbf{z}$, we have that

$$
\begin{array}{r}
\left|\Delta_{2}(t)\right| \leq \alpha\left|\Delta_{1}(t)\right| \leq \alpha \chi_{z}|\mathbf{z}(0)-\gamma(\mathbf{x}(0))| e^{-\left(\lambda_{z} / \epsilon\right) t}+ \\
\frac{\alpha(d+K) \chi_{z}}{\lambda_{z}} \epsilon, \quad \forall t \geq 0 .
\end{array}
$$

Letting $R=\left|\boldsymbol{\Theta}_{x}\left(\mathbf{x}-\mathbf{x}_{\gamma}\right)\right|$, we have that (see [18])

$$
\frac{d}{d t} R+\lambda_{x} R \leq\left|\Theta_{x} \Delta_{2}(t)\right|,
$$

which, given the bound on $\Delta_{2}(t)$, gives

$$
\begin{array}{r}
\frac{d}{d t} R+\lambda_{x} R \leq K_{x} \alpha \chi_{z}|\mathbf{z}(0)-\gamma(\mathbf{x}(0))| e^{-\left(\lambda_{z} / \epsilon\right) t}+ \\
K_{x} \frac{\alpha(d+K) \chi_{z}}{\lambda_{z}} \epsilon,
\end{array}
$$

in which $K_{x}$ is the maximum singular value of $\boldsymbol{\Theta}_{x}$. Let $c_{0}:=\alpha \chi_{z}|\mathbf{z}(0)-\gamma(\mathbf{x}(0))|$ and $c_{1}:=\frac{\alpha(d+K) \chi_{z}}{\lambda_{z}} \epsilon$. Then, equation (12) leads to

$$
\begin{array}{r}
R(t) \leq R(0) e^{-\lambda_{x} t}+\frac{c_{0} K_{x} \epsilon}{\lambda_{z}-\epsilon \lambda_{x}}\left(e^{-\lambda_{x} t}-e^{-\left(\lambda_{z} / \epsilon\right) t}\right)+ \\
\frac{c_{1} K_{x}}{\lambda_{x}}\left(1-e^{-\lambda_{x} t}\right) .
\end{array}
$$

From this equation, using that $R(0) \leq K_{x}\left|\mathbf{x}(0)-\mathbf{x}_{\gamma}(0)\right|$ and that $R(t) \geq K_{x}^{\prime}\left|\mathbf{x}(t)-\mathbf{x}_{\gamma}(t)\right|$, in which $K_{x}^{\prime}$ is the smallest singular value of $\boldsymbol{\Theta}_{x}$, we obtain inequality (10) with $\chi_{x}=$ $K_{x} / K_{x}^{\prime}$ the condition number of $\boldsymbol{\Theta}_{x}$.

Finally, since $\left|\mathbf{z}(t)-\gamma\left(\mathbf{x}_{\gamma}(t)\right)\right| \leq|\mathbf{z}(t)-\gamma(\mathbf{x}(t))|+$ $\left|\gamma(\mathbf{x}(t))-\gamma\left(\mathbf{x}_{\gamma}(t)\right)\right|$ and $\gamma(\mathbf{x})$ is Lipschitz with Lipschitz constant $\alpha_{\gamma}$, inequality (11) follows from Lemma 2 and inequality (10).

Remark 1: In the standard singular perturbation framework, we have that $\mathbf{x}(0)=\mathbf{x}_{\gamma}(0)$ and that $\epsilon \ll 1$. Under these conditions, Theorem 2 implies that for any given $t_{b}>0$, there is an $\epsilon^{*}>0$ such that for all $\epsilon \leq \epsilon^{*}$ we have that $\left|\mathbf{z}(t)-\gamma\left(\mathbf{x}_{\gamma}(t)\right)\right|=O(\epsilon)$ for all $t \geq t_{b}$ and that $\left|\mathbf{x}(t)-\mathbf{x}_{\gamma}(t)\right|=O(\epsilon)$ for all $t \geq 0$. This is consistent with standard singular perturbation results [12]. The advantage of the approach through contraction theory is that $\epsilon$ does not need to be small for the provided bounds to hold and the bounds are quantified exactly in terms of known parameters and $\epsilon$. The conditions required by the contraction approach are, however, stronger than the local exponential stability requirements in standard singular perturbation theory.

Theorem 3: Under the hypotheses of the two previous theorems, denote the overall system's generalized Jacobian by

$$
\mathbf{F}(\mathbf{x}, \mathbf{z}, t)=\left[\begin{array}{ll}
\mathbf{F}_{11} & \mathbf{F}_{12} \\
\mathbf{F}_{21} & \mathbf{F}_{22}
\end{array}\right]
$$


The overall system is contracting if

$$
\sigma_{\max }^{2}\left(\mathbf{F}_{12}+\mathbf{F}_{21}^{T}\right) \leq 4 \lambda_{x} \lambda_{z},
$$

where $\sigma_{\max }$ denotes a uniform upper bound on the largest singular value.

Proof: Use an extra coordinate transformation $\operatorname{diag}\left(\mathbf{I}_{x}, \sqrt{\epsilon} \mathbf{I}_{z}\right)$ and the small-gain theorem [25].

\section{ATTAINING MODULARITY IN BIOMOLECULAR SYSTEMS THROUGH TIMESCALE SEPARATION}

Here, we illustrate how the tools developed in the previous sections can be applied to obtain explicit bounds on retroactivity attenuation as studied in earlier work $[9,10]$. Let $\mathbf{u} \in D_{u} \subset \mathbb{R}_{+}^{q}, \mathbf{y} \in D_{y} \subset \mathbb{R}_{+}^{n}$, and $\mathbf{v} \in D_{v} \subset \mathbb{R}_{+}^{p}$ be vectors whose components denote concentrations of chemical species, such as proteins, enzymes, DNA sites, etc. We consider the following model for an isolated biomolecular system (similar to that of metabolic networks [14]):

$$
\begin{aligned}
& \dot{\mathbf{u}}_{\text {is }}=\mathbf{h}\left(\mathbf{u}_{\text {is }}, t\right)+G_{1} \mathbf{A} \mathbf{r}\left(\mathbf{y}_{\text {is }}, \mathbf{u}_{\text {is }}\right) \\
& \dot{\mathbf{y}}_{\text {is }}=G_{1} \mathbf{B} \mathbf{r}\left(\mathbf{y}_{\text {is }}, \mathbf{u}_{\text {is }}\right)+G_{1} \mathbf{l}\left(\mathbf{y}_{\text {is }}, \mathbf{u}_{\text {is }}\right),
\end{aligned}
$$

in which $\mathbf{r}(\mathbf{y}, \mathbf{u}) \in \mathbb{R}^{r}$ is a reaction rate vector modeling the interaction of species in the vector $\mathbf{u}$ with species in the vector $\mathbf{y}, \mathbf{l}(\mathbf{y}, \mathbf{u}) \in \mathbb{R}^{n}$ is a reaction rate vector driving the dynamics of $\mathbf{y}, \mathbf{A} \in \mathbb{R}^{r \times q}, \mathbf{B} \in \mathbb{R}^{r \times n}$, and $G_{1}$ is a positive constant. Consider next the interconnection of this system with a downstream system whose vector of species is $\mathbf{v}$ :

$$
\begin{aligned}
\dot{\mathbf{u}} & =\mathbf{h}(\mathbf{u}, t)+G_{1} \mathbf{A} \mathbf{r}(\mathbf{y}, \mathbf{u}) \\
\dot{\mathbf{y}} & =G_{1} \mathbf{B} \mathbf{r}(\mathbf{y}, \mathbf{u})+G_{1} \mathbf{l}(\mathbf{y}, \mathbf{u})+G_{2} \mathbf{C s}(\mathbf{y}, \mathbf{v}) \\
\dot{\mathbf{v}} & =G_{2} \mathbf{D} \mathbf{s}(\mathbf{y}, \mathbf{v})
\end{aligned}
$$

in which $\mathbf{s}(\mathbf{y}, \mathbf{v}) \in \mathbb{R}^{s}$ is a reaction rate vector modeling the interaction between the $\mathbf{y}$-subsystem and the $\mathbf{v}$-subsystem. Here, $G_{2}$ is a positive constant such that $G_{2}=\beta G_{1}$ with $\beta>0$. We assume that $\mathbf{u}(0)=\mathbf{u}_{\text {is }}(0)$ and $\mathbf{y}(0)=\mathbf{y}_{\text {is }}(0)$.

System (14) is a general model for a biomolecular system. Interconnections always occur through reactions, whose rates ( $\mathbf{r}$ and $\mathbf{s}$, in this case) appear in both the upstream and the downstream systems with different coefficients (captured by matrices A, B, C, and D). Constant $G_{1}$ models the timescale of the system. We are interested in those cases in which the system evolves on a faster timescale than that of its input, that is, $G_{1} \gg 1$. This situation is encountered, for example, when the $\mathbf{y}$ dynamics model protein modification processes (such as phosphorylation, allosteric modification, dimerization, etc.), while the dynamics of $\mathbf{u}$ model slower processes such as protein production and decay or signaling from outside the cell (here modeled by $\mathbf{h}(\mathbf{u}, t)$ ) [1, 8, 22]. Constant $G_{2}$ models the timescale of the interconnection mechanism of the $\mathbf{y}$-subsystems with the $\mathbf{v}$-subsystem. For example, when this downstream system models gene expression, $\mathbf{s}$ models the binding and unbinding process of transcription factors to DNA binding sites. This reaction is faster than expression and degradation of proteins and therefore we also have that $G_{2} \gg 1[1,6]$.
Definition 2: (Functionally Modular Interconnection) We say that the interconnection of system (14) is functionally modular provided there are constants $K_{0}, K_{1}, \lambda>0$ (not depending on $G_{1}$ and $G_{2}$ ) such that

$$
\left|\mathbf{y}(t)-\mathbf{y}_{\text {is }}(t)\right| \leq K_{0} e^{-\lambda G_{1} t}+\frac{K_{1}}{G_{1}} .
$$

Basically, functional modularity means that if one can increase the gain $G_{1}$, then the interconnection to downstream systems does not affect the output $\mathbf{y}$ of the system. That is, loading effects on the output can be arbitrarily attenuated by increasing gain $G_{1}$. Note that system (14) can be viewed as a perturbed version of system (13). Hence, one could, in principle, apply the robustness result given in Lemma 1. Assuming that the isolated system is contracting with contraction rate $\lambda G_{1}$, one would obtain that the trajectories of the perturbed system exponentially converge with rate $\lambda G_{1}$ to a neighbor of the isolated system trajectory of amplitude $a G_{2} / G_{1}$ for a suitable $a>0$. This would not show that the interconnection is functionally modular because this neighbor cannot be made arbitrarily small by increasing $G_{1}$ given that $G_{2}=\beta G_{1}$. We show in what follows that even if $G_{2}$ is as large as $G_{1}$, the structure of the interconnection and the application of the results of the previous section lead to showing that the interconnection is functionally modular.

In order to proceed, we assume that system (14) has the two following properties (see [10]).

P1 There is an invertible matrix $\mathbf{T} \in \mathbb{R}^{q \times q}$ and a matrix $\mathbf{M} \in \mathbb{R}^{n \times q}$ such that

$$
\begin{aligned}
& \mathbf{T} \mathbf{A}+\mathbf{M} \mathbf{B}=0 ; \\
& \mathbf{M ~} \mathbf{l}(\mathbf{y}, \mathbf{u})=0 \text { for all }(\mathbf{y}, \mathbf{u}) ; \\
& \mathbf{M ~ C}=0 .
\end{aligned}
$$

P2 $\operatorname{ker}(\mathbf{D}) \subseteq \operatorname{ker}(\mathbf{C})$.

Using the change of variables $\mathbf{x}=\mathbf{T} \mathbf{u}+\mathbf{M} \mathbf{y}$ in both isolated and connected systems, using Property P1, and letting $\epsilon=1 / G_{1}$, we obtain

$$
\begin{array}{r}
\dot{\mathbf{x}}_{\text {is }}=\mathbf{T ~} \mathbf{h}\left(\mathbf{T}^{-1}\left(\mathbf{x}_{\text {is }}-\mathbf{M} \mathbf{y}_{\text {is }}\right), t\right) \\
\epsilon \dot{\mathbf{y}}_{\text {is }}=\mathbf{B ~} \mathbf{r}\left(\mathbf{y}_{\text {is }}, \mathbf{T}^{-1}\left(\mathbf{x}_{\text {is }}-\mathbf{M} \mathbf{y}_{\text {is }}\right)\right)+ \\
\mathbf{l}\left(\mathbf{y}_{\text {is }}, \mathbf{T}^{-1}\left(\mathbf{x}_{\text {is }}-\mathbf{M} \mathbf{y}_{\text {is }}\right)\right),
\end{array}
$$

and

$$
\begin{aligned}
\dot{\mathbf{x}}= & \mathbf{T} \mathbf{h}\left(\mathbf{T}^{-1}(\mathbf{x}-\mathbf{M} \mathbf{y}), t\right) \\
\epsilon \dot{\mathbf{y}}= & \mathbf{B} \mathbf{r}\left(\mathbf{y}, \mathbf{T}^{-1}(\mathbf{x}-\mathbf{M} \mathbf{y})\right)+\mathbf{l}(\mathbf{y}, \\
& \left.\mathbf{T}^{-1}(\mathbf{x}-\mathbf{M} \mathbf{y})\right)+\beta \mathbf{C ~ s}(\mathbf{y}, \mathbf{v}) \\
\epsilon \dot{\mathbf{v}}= & \beta \mathbf{D} \mathbf{s}(\mathbf{x}, \mathbf{v}) .
\end{aligned}
$$

In biomolecular systems, these properties are often satisfied because of the physical mechanism of the interconnection. In particular P1 is satisfied because the interconnection occurs through reversible binding, which implies that rates $\mathbf{r}$ will be found with opposite signs in the equation of $\mathbf{u}$ and in the equation of $\mathbf{y}$. Property P2 is also satisfied because all the components of the rate $\mathbf{D}$ s usually appear in the components of the rate $\mathbf{C} \mathbf{s}$. These properties can be easily verified by inspection. 
Lemma 3: Assume that system (15) is partially contracting in $\mathbf{y}_{\text {is }}$, with an associated metric transformation $\boldsymbol{\Theta}_{y}$, and let $\lambda_{y} / \epsilon$ be the contraction rate. Let $\mathbf{y}=\gamma_{y}(\mathbf{x})$ be the globally unique and differentiable solution of

$$
\mathbf{B} \mathbf{r}\left(\mathbf{y}, \mathbf{T}^{-1}(\mathbf{x}-\mathbf{M} \mathbf{y})\right)+\mathbf{l}\left(\mathbf{y}, \mathbf{T}^{-1}(\mathbf{x}-\mathbf{M} \mathbf{y})\right)=0 \text {. }
$$

Assume that there is $d>0$ such that

$$
\forall \mathbf{y}, \forall \mathbf{x}, \forall t \geq 0,\left|\frac{\partial \gamma_{y}(\mathbf{x})}{\partial \mathbf{x}} \mathbf{T} \mathbf{h}\left(\mathbf{T}^{-1}(\mathbf{x}-\mathbf{M} \mathbf{y}), t\right)\right| \leq d
$$

Further, assume that system (15) is partially contracting in $\mathbf{x}_{\text {is }}$, with an associated metric transformation $\boldsymbol{\Theta}_{x}$, and let $\lambda_{x}$ be the contraction rate. Let $\mathbf{T} \mathbf{h}\left(\mathbf{T}^{-1}(\mathbf{x}-\mathbf{M} \mathbf{y}), t\right)$ be Lipschitz in $\mathbf{y}$, with Lipschitz constant $\alpha$ and let $\gamma_{y}(\mathbf{x})$ be Lipschitz with constant $\alpha_{\gamma}$. Let $\mathbf{x}_{\gamma}$ be the solution of the reduced system

$$
\dot{\mathbf{x}}_{\gamma}=\mathbf{T} \mathbf{h}\left(\mathbf{T}^{-1}\left(\mathbf{x}-\mathbf{M} \gamma_{y}(\mathbf{x})\right), t\right), \mathbf{x}_{\gamma}(0)=\mathbf{x}(0) .
$$

Then, for all $t \geq 0$ we have that

$$
\begin{array}{r}
\left|\mathbf{y}_{\text {is }}(t)-\gamma_{y}\left(\mathbf{x}_{\gamma}(t)\right)\right| \leq \chi_{y} e^{-\left(\lambda_{y} / \epsilon\right) t}\left|\mathbf{y}_{\text {is }}(0)-\gamma_{y}(\mathbf{x}(0))\right|+ \\
\frac{d \chi_{y} \epsilon}{\lambda_{y}}+\alpha_{\gamma} \epsilon\left(C_{1}\left(e^{-\lambda_{x} t}-e^{-\left(\lambda_{y} / \epsilon\right) t}\right)+C_{2}\left(1-e^{-\lambda_{x} t}\right)\right),
\end{array}
$$

in which

$$
C_{1}=\frac{\chi_{x} \alpha \chi_{y}\left|\mathbf{y}_{\mathrm{is}}(0)-\gamma_{y}\left(\mathbf{x}_{\mathrm{is}}(0)\right)\right|}{\left(\lambda_{y}-\epsilon \lambda_{x}\right)}, C_{2}=\frac{\chi_{y} \alpha \chi_{x} d}{\lambda_{y} \lambda_{x}},
$$

with $\chi_{y}$ and $\chi_{x}$ upper bounds on the condition numbers of $\boldsymbol{\Theta}_{y}$ and $\boldsymbol{\Theta}_{x}$, respectively.

Proof: Apply Theorem 2 to system (15) with $\mathbf{x}=$ $\mathbf{x}_{\text {is }}, \mathbf{z}=\mathbf{y}_{\text {is }}, \mathbf{f}(\mathbf{x}, \mathbf{z}, t)=\mathbf{T} \mathbf{h}\left(\mathbf{T}^{-1}\left(\mathbf{x}_{\text {is }}-\mathbf{M} \mathbf{y}_{\text {is }}\right)\right)$, and $\mathbf{g}(\mathbf{x}, \mathbf{z}, \epsilon)=\mathbf{B} \mathbf{r}\left(\mathbf{y}_{\text {is }}, \mathbf{T}^{-1}\left(\mathbf{x}_{\text {is }}-\mathbf{M} \mathbf{y}_{\text {is }}\right)\right)+\mathbf{l}\left(\mathbf{y}_{\text {is }}, \mathbf{T}^{-1}\left(\mathbf{x}_{\text {is }}-\right.\right.$ $\left.\left.\mathbf{M} \mathbf{y}_{\text {is }}\right)\right)$.

Lemma 4: Let the assumptions of Lemma 3 be satisfied. Let system (16) be partially contracting in $\mathbf{z}=(\mathbf{y}, \mathbf{v})$ with associated metric transformation $\Theta_{z}$ and let $\lambda_{z} / \epsilon$ be the contraction rate. Let $\mathbf{z}=\gamma_{z}(\mathbf{x})$ be the globally unique solution of

$$
\begin{gathered}
\operatorname{Ds}(\mathbf{x}, \mathbf{v})=0 \\
\mathbf{B r}\left(\mathbf{y}, \mathbf{T}^{-1}(\mathbf{x}-\mathbf{M y})\right)+\mathbf{l}\left(\mathbf{y}, \mathbf{T}^{-1}(\mathbf{x}-\mathbf{M y})\right)+\beta \mathbf{C s}(\mathbf{y}, \mathbf{v})=0 .
\end{gathered}
$$

Assume that there is $d^{\prime}>0$ such that

$$
\forall \mathbf{z}, \forall \mathbf{x}, \forall t \geq 0, \quad\left|\frac{\partial \gamma_{z}(\mathbf{x})}{\partial \mathbf{x}} \mathbf{T h}\left(\mathbf{T}^{-1}(\mathbf{x}-\mathbf{M} \mathbf{y}), t\right)\right| \leq d^{\prime}
$$

Let $\gamma_{z}(\mathbf{x})$ be Lipschitz with constant $\alpha_{\gamma}^{\prime}$. Let $x_{\gamma}$ be the solution of the reduced system (18). Then, for all $t \geq 0$ we have that

$$
\begin{array}{r}
\left|\mathbf{y}(t)-\gamma_{y}\left(\mathbf{x}_{\gamma}(t)\right)\right| \leq \chi_{z} e^{-\left(\lambda_{z} / \epsilon\right) t}|\mathbf{z}(0)-\gamma(\mathbf{z}(0))|+ \\
\frac{d \chi_{z} \epsilon}{\lambda_{z}}+\alpha_{\gamma}^{\prime} \epsilon\left(C_{1}^{\prime}\left(e^{-\lambda_{x} t}-e^{-\left(\lambda_{z} / \epsilon\right) t}\right)+C_{2}^{\prime}\left(1-e^{-\lambda_{x} t}\right)\right),
\end{array}
$$

in which

$$
C_{1}^{\prime}=\frac{\chi_{z} \alpha \chi_{x}\left|\mathbf{z}(0)-\gamma_{z}(\mathbf{x}(0))\right|}{\left(\lambda_{z}-\epsilon \lambda_{x}\right)}, \quad C_{2}^{\prime}=\frac{\chi_{z} \alpha \chi_{x} d^{\prime}}{\lambda_{z} \lambda_{x}},
$$

with $\chi_{z}$ and $\chi_{x}$ upper bounds on the condition numbers of $\boldsymbol{\Theta}_{z}$ and $\boldsymbol{\Theta}_{x}$, respectively.
Proof: Apply Theorem 2 with $\mathbf{f}(\mathbf{x}, \mathbf{z}, t)=$ $\mathbf{T} \mathbf{h}\left(\mathbf{T}^{-1}(\mathbf{x}-\mathbf{M} \mathbf{y}), t\right), \mathbf{g}(\mathbf{x}, \mathbf{z}, \epsilon)=\left(\mathbf{B r}\left(\mathbf{y}, \mathbf{T}^{-1}(\mathbf{x}-\right.\right.$ $\left.\mathbf{M y}))+\mathbf{l}\left(\mathbf{y}, \mathbf{T}^{-1}(\mathbf{x}-\mathbf{M} \mathbf{y})\right)+\beta \mathbf{C s}(\mathbf{y}, \mathbf{v}), \beta \mathbf{D} \mathbf{s}(\mathbf{y}, \mathbf{v})\right)$ and take into account that $\mathbf{D s}(\mathbf{y}, \mathbf{v})=0$ implies $\mathbf{C s}(\mathbf{y}, \mathbf{v})=0$ by Property P2 so that $\gamma_{z}(\mathbf{x})=\left(\gamma_{y}(\mathbf{x}), \gamma_{v}(\mathbf{x})\right)$ with $\gamma_{y}(\mathbf{x})$ as in Lemma 3.

Theorem 4: Let the assumptions of Lemma 3 and Lemma 4 hold. Then, the interconnected system (14) satisfies the modular interconnection property.

Proof: It follows from Lemma 3, Lemma 4, and the triangular inequality.

\section{EXAMPLE}

As an example, we consider a phosphorylation cycle and demonstrate that the interconnection to downstream targets is modular. For simplicity, we consider a one-step reaction model for phosphorylation. For any species X, we denote by $X$ (italics) its concentration. Let $\mathrm{Z}$ be a kinase expressed at (time-varying) rate $k(t)$ and degraded at rate $\delta$. Let its substrate be $\mathrm{X}$ and let $\mathrm{X}^{*}$ denote the phosphorylated version of $\mathrm{X}$. Let the total amount of $\mathrm{X}$ be constant and denoted by $X_{T}$. Let $Y$ be the phosphatase in total amount $Y_{T}$. Then, the phosphorylation reactions are given by $\mathrm{Z}+\mathrm{X} \stackrel{k_{1}}{\longrightarrow}$ $\mathrm{X}^{*}+\mathrm{Z}, \quad \mathrm{Y}+\mathrm{X}^{*} \stackrel{k_{2}}{\longrightarrow} \mathrm{X}+\mathrm{Y}$ and the binding reaction with downstream targets $\mathrm{p}$ is given by $\mathrm{X}^{*}+\mathrm{p} \underset{k_{\text {off }}}{\stackrel{k_{\mathrm{on}}}{\rightleftharpoons}} \mathrm{C}$. We denote the total concentration of downstream targets by $p_{T}$. With conservation laws $p_{T}=C+p, X_{T}=X^{*}+X+C$, and assuming that $p_{T} / X_{T} \ll 1$, we have the following expression for the isolated system

$$
\begin{aligned}
\dot{Z} & =k(t)-\delta Z \\
\dot{X}^{*} & =k_{1} X_{T} Z\left(1-\frac{X^{*}}{X_{T}}\right)-k_{2} Y_{T} X^{*},
\end{aligned}
$$

and for the interconnection

$$
\begin{aligned}
\dot{Z}= & k(t)-\delta Z \\
\dot{X}^{*}= & k_{1} X_{T} Z\left(1-\frac{X^{*}}{X_{T}}\right)-k_{2} Y_{T} X^{*} \\
& -k_{\mathrm{on}} X^{*}\left(p_{T}-C\right)+k_{\mathrm{off}} C \\
\dot{C}= & k_{\mathrm{on}} X^{*}\left(p_{T}-C\right)-k_{\mathrm{off}} C .
\end{aligned}
$$

In this system, we have $k_{1}, k_{2}, k_{\text {on }}, k_{\text {off }} \gg \delta, k(t)$. Define $G_{1}:=k_{1} X_{T}$ and let $\alpha:=k_{2} / G_{1}$. Define $G_{2}:=k_{\text {on }}$ and let $k_{d}:=k_{\text {off }} / G_{2}$. Letting also $u:=Z, y:=X^{*}$, and $v:=C$, the isolated system can be re-written as

$$
\begin{aligned}
\dot{u} & =k(t)-\delta u \\
\dot{y} & =G_{1}\left(u\left(1-\frac{y}{X_{T}}\right)-\alpha Y_{T} y\right),
\end{aligned}
$$

and the interconnection with downstream targets is given by

$$
\begin{aligned}
\dot{u}= & k(t)-\delta u \\
\dot{y}= & G_{1}\left(u\left(1-\frac{y}{X_{T}}\right)-\alpha Y_{T} y\right) \\
& -G_{2}\left(y\left(p_{T}-v\right)-k_{d} v\right) \\
\dot{v}= & G_{2}\left(y\left(p_{T}-v\right)-k_{d} v\right),
\end{aligned}
$$


which are in the forms (13)-(14) with

$$
\begin{gathered}
r(y, u)=0, h(u, t)=k(t)-\delta u, \\
l(y, u)=\left(u\left(1-\frac{y}{X_{T}}\right)-\alpha Y_{T} y\right), \\
s(y, v)=y\left(p_{T}-v\right)-k_{d} v .
\end{gathered}
$$

Note that system (19) is already in the form (15) and system (20) is already in the form (16) with $\epsilon=1 / G_{1}$ and $G_{2}=$ $\beta G_{1}$. Hence, we can take $T=\mathbb{I}$ and $M=0$. One can easily verify the assumptions of Theorem 4 . In particular, the isolated system is partially contracting in $\mathbf{z}=y$ and in $\mathbf{x}=u$ with $\boldsymbol{\Theta}_{x}=\boldsymbol{\Theta}_{y}=\mathbb{I}$. The function $\mathbf{f}(\mathbf{x}, \mathbf{z}, t)=k(t)-\delta u$ is Lipschitz and $\gamma_{y}(u)$ is given by $\gamma_{y}(u)=\frac{u}{\left(\left(u / X_{T}\right)+\alpha Y_{T}\right)}$, which is globally defined for $u \in \mathbb{R}_{+}$, differentiable, and Lipschitz. Assuming that $k(t)$ is bounded, we also have that $\left|\frac{\partial \gamma_{y}(u)}{\partial u} \mathbf{f}(\mathbf{x}, \mathbf{z}, t)\right|<d$ for some suitable $d>0$.

The connected system is partially contracting in $\mathbf{z}=$ $(y, v)$ with measure $m_{2}$ and $\boldsymbol{\Theta}_{z}=\mathbb{I}$. This can be seen by computing the eigenvalues of the symmetric part of the Jacobian $\mathbf{J}$ given by

$$
J=\left(\begin{array}{cc}
-\left(u / X_{T}\right)-\alpha Y_{T}-\beta\left(p_{T}-v\right) & \beta y+\beta k_{d} \\
\beta\left(p_{T}-v\right) & -\beta y-\beta k_{d}
\end{array}\right) .
$$

Denoting $a:=\left(u / X_{T}\right)+\alpha Y_{T}, b=\beta\left(p_{T}-v\right)$, and $c=\beta\left(y+k_{d}\right)$, we have that the eigenvalues of $\frac{1}{2}(J+$ $\left.J^{T}\right)$ are negative provided $\operatorname{det}\left(\frac{1}{2}\left(J+J^{T}\right)\right)>0$, which is satisfied whenever $4 c(a+b)>(c+b)^{2}$. This is, in turn, satisfied if $a$ is sufficiently large, which can be guaranteed by taking $Y_{T}$ sufficiently large. Furthermore, we have that $\gamma_{z}(u)=\left(\frac{u}{\left(u / X_{T}+\alpha Y_{T}\right)}, \frac{p_{T} y}{y+k_{d}}\right)$, with $y=\frac{u}{u / X_{T}+\alpha Y_{T}}$, which is uniquely defined, differentiable everywhere and globally Lipschitz for $u \in \mathbb{R}_{+}$. Assuming that $k(t)$ is bounded, we also have that $\left|\frac{\partial \gamma_{z}(u)}{\partial u} \mathbf{f}(\mathbf{x}, \mathbf{z}, t)\right|$ is uniformly bounded.

Hence, we conclude that the system satisfies the modular interconnection property as the assumptions of Theorem 4 are satisfied. In particular, the difference between the isolated and connected system behavior can be rendered smaller by increasing the values of $G_{1}$, which can be performed by increasing the amounts of total substrate $X_{T}$. Furthermore, to guarantee contraction of the interconnection, one needs to guarantee a large enough, which can be obtained by employing sufficiently large amounts of phosphatase $Y_{T}$. In turn, large amounts of substrate and phosphatase in phosphorylation cycles have been shown to be at the basis of a fundamental principle for insulation from retroactivity $[5,10]$.

\section{CONCLUSIONS}

In this paper, we have revisited standard singular perturbation results by employing tools from contraction theory. Assuming that the fast and slow subsystems are each partially contracting, we obtained a global result about the convergence of the system trajectories to the slow manifold on the infinite time interval. Furthermore, explicit bounds on the convergence rate and on the asymptotic error between the trajectories of the singularly perturbed system and the reduced system were obtained. These results were applied to obtain explicit bounds and a global result on retroactivity attenuation in biomolecular systems.

\section{REFERENCES}

[1] U. Alon. An introduction to systems biology. Design principles of biological circuits. Chapman-Hall, 2007.

[2] E. Andrianantoandro, S. Basu, D. K. Karig, and R. Weiss. Synthetic biology: New engineering rules for an emerging discipline. Molecular Systems Biology, 2:1-14, 2006.

[3] D. Angeli. A Lyapunov approach to incremental stability properties. IEEE Transactions on Automatic Control, 47:410-321, 2002.

[4] G. Dahlquist. Stability and error bounds in the numerical integration of ordinary differential equations. Transanctions of the Royal Institute Technology (Stockholm), 1959.

[5] D. Del Vecchio, A. J. Ninfa, and E. D. Sontag. Modular cell biology: Retroactivity and insulation. Molecular Systems Biology, 4:161, 2008.

[6] Gordon L. Hager and Akhilesh K. Nagaich. Transcription factor dynamics. In Jun Ma, editor, Gene Expression and Regulation, chapter 30, pages 493-502. Springer New York, New York, NY, 2006.

[7] P. Hartman. On stability in the large for systems of ordinary differential equations. Canadian Journal of Mathematics, 13:480-492, 1961.

[8] J. J. Hornberg, B. Binder, F. J. Bruggeman, B. Schoeber, R. Heinrich, and H. V. Westerhoff. Control of MAPK signaling: from complexity to what really matters. Oncogene, 24:5533-5542, 2005.

[9] S. Jayanthi and D. Del Vecchio. Retroactivity attenuation in transcriptional networks: Design and analysis of an insulation device. Proc. IEEE Conf. Decision and Control, pages 774-780, 2008.

[10] S. Jayanthi and D. Del Vecchio. Retroactivity attenuation in biomolecular systems based on timescale separation. IEEE Trans. Automatic Control, 56(4):748-761, 2010.

[11] N. Kashtan and U. Alon. Spontaneous evolution of modularity and network motifs. Proc. of the Natl. Acad. of Sci., 39:13773-13778, 2005.

[12] H. Khalil. Nonlinear Systems. Prentice Hall, 2002.

[13] M. W. Kirschner and J. C. Gerhart. The Plausibility of Life: Resolving Darwin's Dilemma. Yale University Press, 2005.

[14] E. Klipp, R. Herwig, A. Kowald, C. Wierling, and H. Lehrach. Systems Biology in Practice. Wiley-VCH, 2005.

[15] P. Kokotovic, H. K. Khalil, and J. O'Reilly. Singular Perturbation Methods in Control. SIAM, 1999.

[16] D. C. Lewis. Metric properties of differential equations. American Journal of Mathematics, 71:294-312, 1949.

[17] W. Lohmiller and J. J. Slotine. Contraction analysis of non-linear distributed systems. International Journal of Control, 78:678-688, 2005.

[18] W. Lohmiller and J. J. E. Slotine. On contraction analysis for nonlinear systems. Automatica, 34:683-696, 1998.

[19] W. Lohmiller and J.J.E Slotine. Nonlinear process control using contraction theory. A.I.Ch.E. Journal, 2000.

[20] S. M. Lozinskii. Error estimate for numerical integration of ordinary differential equations. I. Izv. Vtssh. Uchebn. Zaved Matematika, 5:222222, 1959.

[21] A. Pavlov, A. Pogromvsky, N. van de Wouv, and H. Nijmeijer. Convergent dynamics, a tribute to Boris Pavlovich Demidovich. Systems and Control Letters, 52:257-261, 2004.

[22] J. M. Rohwer, N. D. Meadow, S. Roseman, H. V. Westerhoff, and P. W. Postma. Understanding glucose transport by the bacterial phosphoenolpyruvate: glucose phosphotransferase system on the basis of kinetic measurements in vitro. The Journal of biological chemistry, 275(45):34909-34921, November 2000.

[23] Herbert A. Simon. The architecture of complexity. Proceedings of the American Philosophical Society, 6:467-482, 1962.

[24] J.J. Slotine. Modular stability tools for distributed computation and control. International Journal of Adaptive Control and Signal Processing, 17:397-416, 2003.

[25] W. Wang and J. J. E. Slotine. On partial contraction analysis for coupled nonlinear oscillators. Biological Cybernetics, 92:38-53, 2005. 